\title{
Dieu en tant que bien suprême selon I'acception de St. Augustin
}

L'idée de Dieu en tant que Bien Suprême a été posée par St. Augustin comme clef de tout son système. Elle se trouve à la base de la théologie, de la métaphysique, de l'épistémologie, de l'anthropologie et de l'éthique. Le Bien Suprême est à l'origine de tout, et en particulier du libre arbitre de l'homme et de ses bonnes actions. $\mathrm{Ce}$ Bien est à la fois le but suprême vers lequel tout chemine par amour. Le Summum Bonum est "la vie des âmes, la vie des vies" ". Le Summum Bonum augustinien peut être considéré sous deux aspects : celui de sa nature intérieure et celui en relation aux biens accidentels. Ce dernier aspect peut être examiné également soit en sa genèse primordiale, soit du point de vue de l'actuelle protection divine (problème de la Providence). Ce problème-ci, englobant le thème du sens du mal physique et moral, est entièrement absent de la dissertation présente.

St. Augustin attribuait à Dieu de nombreux noms, parmi lesquels il préfère expréssement le terme Bonum, éventuellement les appellations synonymes comme: Summum Bonum, Bonum omnis boni, Summa Bonitas, Bonum increatum. Ces noms présentent des nuances de signification différentes, mais ils affirment toujours l'identité de la nature de Dieu au bien existant et moral. L'argument au moyen duquel l'évêque d'Hippone prouve l'existence du Bien Suprême, a été examiné précédemment ${ }^{2}$. A présent, nous allons considérer son as-

1. Confessions $3,6,10$. Bibliothèque Augustinienne. Oeuvres de St. Augustin, t. XIII. Trad. de E. Tréhorel et G. Bouissou, Paris 1962, Desclée de Brouwer, p. 383. Nous citerons les oeuvres de St. Augustin d'après: Bibliothèque Augustinienne, BA; Migne, Patrología Latina, t. 32-47, Pa ris 1861-1862.

2. Voir: KOWALCZYK S., L'argument axiologique dans la philosophie de Dieu de St. Augustin, "Estudio Agustiniano", 5 (1970), 51-59. 
pect statique (attributs et structure) et dynamique (causalité efficiente, exemplaire, finale).

\section{Nature intérieure du Bien Suprême}

Maintes fois, le penseur chrétien antique affirmait: "Deus summe bonus est, quia summe est qui sit, Ego sum qui sum) ${ }^{3}$. Le sens de l'énoncé est clair: le bien ontique de la nature de Dieu est lié génétiquement à la plénitude d'existence. Dieu est summum esse, les créatures possèdent minus esse. Cette terminologie a un caractère quantitatif, expliqué par certains auteurs par l'influence du néo-platonisme, et en particulier celle de la théorie d'emanation ${ }^{4}$. Il es difficile cependant d'être d'accord avec cette interprétation, puisque $S t$. Augustin avait conscience $d u$ fait que Dieu seul est (habens in se ut sit) ${ }^{5}$. Dieu n'est pas somme des existences dérivées, mais Il est existence autonome. "Le souverain bien, au-dessus duquel il n'y a rien, c'est Dieu, et par là c'est un bien immuable, donc vraiment éternel et vraiment immortel. Tous les autres biens ne sont que par lui mais ne sont de lui) ". L'auteur du traité De natura boni apercevait les différences essentielles entre le Bien Suprême et les autres biens, ces différences étant exprimées par les relations: existence en soi -dérivation, immutabilité - mutabilité, simplicité - complexité.

La conception augustinienne du bien a un caractère expressément qualitatif. Le bien d'une chose concrète ne se réduit pas à ses dimensions quantitatives, mais il réside en sa forme et son existence réelle. Chaque être possédant sa forme propre, ses perfections, sa teneur générique, possède son bien ". Celui-ci a un caractère qualitatif, non quantitatif, utilitaire ou conventionnel.

La différence entre le Bien Suprême et le bien créé n'a non plus de caractère quantitatif, mais elle a un caractère qualitatif. Le Summum Bonum n'est pas uniquement la so $\mathrm{m} \mathrm{m}$ e des biens accidentels. Dieu est un bien de nature exceptionnelle, parce que, dans Son essence, Il comporte l'existence. La plénitude de l'existence divine com-

3. Sermo 156, 6 PL 38, 853. Voir: VON RINTELEN, FR.- J., Dequs bonum omnis boni: "Aurelius Augustinus", Köln 1930, 203-224; VON RINTELEN, FR.- J., Finitud $e$ infinitud del bien en san Agustín. "Augustinus", 13 (1968), 369-383.

4. A. DORNER, Augustinus. Sein theologisches System und seine religionsphilosophische Anschauung, Berlin 1873, 40 ss, 329 ss.

5. De Gen. ad litt. 5, 16, 34 PL 34, 333. Voir: De lib. arb. 1, 2-5 PL 32, 1224-1228.

6. De natura boni 1,1 BA, t. I. Trad. par B. Roland-Gosselin, Paris 1949, 441; PL 42, 440.

7. Voir: Enar. in ps. 102,8 PL 37, 1322; Confes. 7, 12, 18 PL 32, 743. 
prend une autonomie sans entraves et la nécessité d'exister. C'est ainsi que le Summum Bonum est un Bien qui, en excluant de sa nature toute imperfection, et avant tout le néant, doit sa richesse uniquement à soi-même. "Tout est bon, mais tout a été crée par Dieu, non par soi. Celui qui l'a fait est bon au-dessus de tout, puisque personne ne l'a fait bon, mais Il est bon grâce à Lui-même)" ${ }^{8}$. Le bien divin est un bien intégral, nécessaire, exclusivement propre à Lui. Par contre, le bien des créatures est un bien ('d'autrui), c'est-a-dire participé, non-autonome. La cause de cela réside dans le fait que chaque bien mutable, tout en provenant du Bien Suprême, et en lui ressemblant, a été créé à partir du non-être, du néant, ex nihilo, et il y est intérieurement (mêlé).

Le deuxième attribut du Bien intégral est son immutabilité. Dans l'interprétation de St. Augustine, cet attribut est plus essentiel que l'existence autonome. C'est justement par l'immutabilité absolue que s'exprime la plénitude de l'existence de Dieu. "Il est en toute vérité, en effet, parce qu'il est immutable. Car tout mutation anéantit ce qui était. Celui-là donc est en toute vérité qui est immutable) ${ }^{9}$. L'identification de la plénitude de l'être avec l'entière immutabilité se projette -indirectement- sur le caractère du Bien absolu. Selon Augustin, la valeur ontique du Summum Bonum réside avant tout dans l'immutabilité absolue de la nature de Dieu. Ainsi, Bien Suprême est uniquement cette nature non soumise aux mutations quelles qu'elles soient: ni aux mutations substantielles (naissance, mort), ni aux variations accidentelles (quantitatives au qualitatives) ${ }^{10}$. Le Bien de Dieu est un bien permanent, immortel, éternel ${ }^{11}$. Par son caractère, il présente un contraste avec la variabilité continuelle des biens accidentels qui sont soit entièrement périssables (valeurs matérielles), soit pour les moins (amoindrissables) (valeurs spirituelles).

L'attribut suivant du Summum Bonum est sa simplicité. Une nature absolument simple se caractérise par l'identité réelle et pleine entre le sujet et ses propriétés ${ }^{12}$. Ni en récipient rempli de liquide, ni

\footnotetext{
8. Sermo 19, 1, 1 PL 38, 185. Voir: "Bonum quod summe et per se bonum est, et non participatione alicuius boni, sed propria natura et essentia" De mor. Man. 2, 4, 6 PL 32, 1347.

9. De $n$. boni 1, 19 BA, t. I, 457; PL 42, 557. Voir: E. ZUM BRUNN, “L'immutabillté de Dieu selon saint Augustin: "Nova et Vetera" 41 (1966), 219-225.

10. Voir: De lib. arb. 2, 26, 44 PL 32, 1264; Trin. 5, 1, 2 PL 42, 912.

11. "Summum bonum, quo superius non est, Deus est: ac per hoc incommutabile bonum est, ideo vere aeternum, et vere immortale" /De n. boni 1,1 PL 42, 551/; "O bona Domini dulcia, immortalia, incomparabilia, sempiterna, incommutabilia" Enar. in ps. 26, 22 PL 36, 210.

12. Voir: Trin. $8,3,4-5$ PL 42, 949-950.
} 
un homme à formation polymathique ne possèdent pareille simplicité. L'intellect de l'homme n'est pas identique à la science acquise, puisqu'il acquiert sans cesse nouvelles connaissances ou perd celles qu'il posséde.

L'être simple n'est pas divisé en substance et en choses accidentelles. Tous les attributs d'un tel être appartiennent à sa nature. Une pareille unité appartient uniquement au Bien Suprême dans lequel toute propriété s'identifie à la nature. Aucun attribut du Bien intégral ne peut être anéanti ni amoindri ni agrandi ${ }^{13}$. Ainsi, il est impossible que Dieu soit à la fois esprit quant à la substance, et bien quant â la qualité, mais, en Sa nature, Il comprend l'un et l'autre ${ }^{14}$. $\AA$ proprement parler, ce n'est pas que Dieu soit bon, mais le bien intégral et pur est Dieu. Toute Sa nature s'identifie entièrement avec la bonté et diffère des créatures, chez qui le bien, quique organiquement lié à l'existence, est toujours participé ${ }^{15}$. La simplicité du Bien Suprême se manifeste également par ce qu'il est l'être unique chez qui le bien ontique correspond strictement au bien moral ${ }^{16}$. L'accidentalité des créatures apparaît en ce que souvent, le bien moral ne suit pas leur bien ontique. C'est pourorquoi ils est possible qu'existe un homme éthiquement mauvais qui, simultanément, et en tant que nature raisonnable, soit un bien ontique.

Dieu est une nature absolument simple, ainsi il est difficile de parler d'éléments constitutifs du Bien Suprême. On peut cependant se demander, en quoi St. Augustin voyait la richesse essentielle du Bien absolu. Or, c'est avant tout dans l'existence, dans son autonomie, sa plénitude, sa nécessité. L'accentuation du rôle de l'existence dans la nature du Summum Bonum n'a pas été faite sous l'influence du néo-platonisme (Plotin avait pourtant exclu l' existentialité de l'Unité), mais grâce à la lecture du livre biblique de l'Exode. Dieu, en parlant à Moïse, se nomma: "Je suis Celui qui est) (Exode 3, I4) ${ }^{17}$. Il existe actuellement de nombreuses interprétations de cette parole divine, toutefois St. Augustin la comprenait d'une manière existentielle. Son commentaire à l'Evangile de St. Jean le prouve ${ }^{18}$. Il y analyse

\footnotetext{
13. Enar. in ps. 101, $10 \mathrm{PL} 37,1311$.

14. Trin. 8, 3, $4 \mathrm{PL} 42,949$.

15. Le bien simple est le "bien lui-même" /ipsum bonum/, tandis que le bien créé est un bien complexe, "adjoint" à la nature des choses. Voir: Enar. in ps. 26, 8 PL 36, 203.

16. Voir: Trin. $15,5,8 \mathrm{PL} 42,1062-1063$.

17. Voir: C. SIMON PRADO, Praelectlones blblicae, Vetus Testamentum, t. I, Roma 1956, 202-203; Bibel-Lexicon, Herausgegeben von H. Haag, Zürich-Köln 1956, 766-769.

18. In Joan. ev. tr. 38, 8-9 PL 35, 1679-1680; E. GILSON. Le Thomisme, Paris 1947, 123-128.
} 
l'appelation "Qui est) en l'opposant à d'autres comme: Dieu, Créateur, Protecteur, Père etc. Ces appellations, bien que justes, indiquent cependant des relations extérieures et non indispensables de Dieu à l'égard du monde. Par contre, l'appellation "Qui est) ((iipsum Esse») indique la nature intérieure de Dieu. Les attibuts de créateur, de protecteur ou de Père miséricordieux, sont très essentiels, mais il le sont uniquement par rapport à l'homme ${ }^{19}$. L'appellation biblique «ipsum esse) indique ce qui est réservé uniquement à Dieu: la nécessité de l'existence et son autonomie.

La définition existentielle de la nature du Bien Suprême résultant de l'influence du néo-platonisme, gravite, chez St. Augustin, vers un essentialisme modéré. Sans aucun doute, il attribuait à Dieu le caractère de (aseitas), mais il l'interprétait souvent comme (immutabilitas》. Le Bien Suprême est "l'existence-même», parce qu'il exclue de sa nature tout changement, toute mutation aussi bien actuelle que potentielle ${ }^{20}$. En une telle acception, (ipsum esse) indique plutôt la permanence de la nature divine que l'autonomie de l'existence. L'essentialisme métaphysique signalé ci-dessus, n'empêche pas la fait qu'Augustin considérait la plénitude de l'existence de Dieu comme élément intégral du Summum Bonum.

Une autre source de richesse du Summum Bonum est l'caeterna et incommutabilis Forma) ${ }^{21}$. La notion de forme dans êtres créés désigne en élément organisateur et spécificateur, mais par rapport à Dieu, elle possède uniquement un sens analogique. En ce cas, elle est synonyme de perfection. Dieu est Summum Bonum aussi bien au sens extensif qu'intensif. Nous intéresserons d'abord au domaine de richesse de ce Bien.

La conception augustinienne de Dieu diffère essentiellement de la conception néo-platonicienne de l'Unité. Plotin a exclu de l'Unité l'existentiel, le corporel, la pensée, le conscient et la vie, tandis qu' Augustin exclut de la nature de Dieu uniquement ce qui est lié au corporel. Dieu possède donc également des attributs négatifs et des

\footnotetext{
19. Sermo 7,7 PL 38,66 .

20. Voir: Trin. $5,2,3 \mathrm{PL} 42,912 ; \mathrm{H}$. IZUMU, The Notion of Mutabilitas in Augustine's Philosophy: "Studies in medieval Thought", 4 (1961), 102-121; J. F. ANDERSON, S. Augustine and Being, The Hague 1965, passim.

21. "Esse aliquam formam aeternam et incommutabilem, quae neque contineatur et quasi diffundatur, neque potendatur atque varietur temporibus" (De IIb. arb. 2, 26, 44 PL 32, 1264 ).
} 
attributs positifs. Parmi les premiers, l'évêque d'Hippone compta l'immuabilité, l'ilumination dans le temps et dans l'immatérialité, l'espace, l'infinité et l'invisibilité. Il mentionne les attributs positifs de Dieu dans les Soliloquia, en y classant: la vérité, la sagesse, la vie, la bonheur, le bien, le beau, la lumière ${ }^{22}$. Les Confessions donnent une énumération plus abondante des attributs de Dieu; Augustin y fait figure non pas de systématicien mais de fervent chrétien plein d'amour de Dieu ${ }^{23}$. Il y expose les attributs relatifs (concernant les liens entre Dieu et le monde) et les propriétés morales. Une énumération systématique des attributs de Dieu se trouve dans $D e$ Trinitate. On y lit que Dieu est: éternel, immortel, immutable, vivant, sage, fort, beau, juste, bon, spirituel, donnant le bonheur ${ }^{24}$.

La richesse des domaines de la perfection ne présente pas de differentia specifica Bien Suprême, puisque certains attributs de Dieu se réalisent également - toutes proportions gardées- dans la nature humaine. Le caractère differentiel pour le Summum Bonum est sa "qualité») liée à son mode d'existence. Il en résulte que le Bien Suprême est autonome, immutable, et simple. Ce sont ces derniers attributs qui forment la ligne de démarcation entre le bien de Dieu et celui des créatures.

St. Augustin, en caractérisant Dieu, se reporte souvent aux paroles de St. Jean l'Evangéliste: Deus caritas est. La charité n'est pas l'un des attributs de Dieu, elle constitue la synthèse de tous les attributs: "La substance même est charité, la charite même est substance) ${ }^{25}$. La charité est la base de toutes les perfections ${ }^{26}$. Si donc le Summum Bonum est l'unité intégrale des perfections ontiques et éthiques, existant au degré maximum, par cela même il est Charité. Le Summum Bonum est la charité personnifiée et présentant le but final des créatures raisonnables.

2. Le Bien Suprême en tant que cause efficiente, exemplaire, finale.

Le Summum Bonum, pour St. Augustin, est en même temps

\footnotetext{
22. Sol. 1, 1, 3 PL 32,870 .

23. "Quid es ergo, Deus meus? [...] Summe, optime, potentissime, omnipotentissime, misericordissime et iustissime, secretissime et praesentissime, pulcherrime et fortissime, stabilís et incomprehensibilis; immutabilis, mutans omnia; numquam novus, numquam vetus; innovans omnia $[\ldots]$ semper agens, semper quietus, $\lceil\ldots]$ creans et nutriens, et perficiens" (Confes. 1 , 4, 4. PL 32, 662).

24. Trin. $15,5,8$ PL 42,1062

25. Trin. 15, 17, 29 BA, t. XVI. Trad. par P. Agaësse, Paris 1955, 505; PL 42, 1081. Voir: ibidem $8,8,12$ PL 42, 958.

26. Voir: "Charitas est radix bonorum" (De gr. Chr. 1, 20 PL. 44, 370).
} 
Bonum omnis boni. Bonum est diffusivum sui. Ce principe est d'autant plus valable par rapport à Dieu comme bien absolu, cause: a) efficiente, b) exemplaire, et c) finale de tout bien.

a. L'évêque d'Hippone a lié l'activité efficiente, créatrice de Dieu à l'ordre axiologique; il le dit expressément dans De natura bo$n i^{27}$. Cet énoncé témoigne que l'auteur liait l'acte créateur simultanément à l'ordre de l'être et du bien. Dieu, grâce à un seul et même acte de volition, est la cause créatrice de tout être et de tout bien.

Le Bien Suprême est le cause créatrice de tous les biens. Augustin accentue expressément les différences subsistant entre l'acception chrétienne de l'acte créateur et l'émanationnisme néoplatonicien. Dans l'acception de Plotin, l'Unité divise sa nature et sa force propres entre les biens dérivés; dans l'acception du penseur chrétien, Dieu crée le monde. Seul le Verbe éternel est ex Deo, c'est-à-dire il participe à la nature divine; tout le reste cependant n'est que a Deo ${ }^{28}$. "Tous les autres biens ne sont que par lui, mais ils ne sont pas de lui» ${ }^{29}$. Le bien créé est donc oeuvre de l'omnipotence de Dieu mais ne fait pas partie de la nature de l'Absolu et n'est pas son émanation. Plotin acceptait l'existence d'étapes succesives au cours desquelles apparaissaient tour à tour les divers êtres. Augustin accepte l'universalité de l'acte créateur: l'existence et le bien de toutes les créatures provient finalement de Dieu ${ }^{30}$.

L'auteur du De natura boni constate que Dieu (ex nihilo fecit) tous les êtres. En construisant une maison, l'homme se sert de matériaux appropriés auquels il donne la forme qu'il se propose. Par contre, Dieu appelant l'univers à l'existence, ne disposait d'aucun matériau ni d'aucune matiére. L'évêque d'Hippone souligne expressément de ne pas concevoir le néant comme une préexistence ${ }^{31}$. Le néant n'est ni substratum ni matière, car il est exactement le manque de matière réelle, il est (non-existence). L'action créatrice, causale du Bien Suprême diffère donc essentiellement de la fonction du Dé-

27. “Le souverain bien, au-dessus duquel il n'y a rien, c'est Dieu, et par là c'est un bien immuable, donc vraiment éternel et vraiment immortel. Tous les autres biens ne sont que par lui mais ne sont pas de lui". De natura boni 1, 1 BA, t. I, 441; PL 42, 551.

28. "Viennent 'de lui' cela n'a pas la mếme siginification que sont 'de lui'". De natura boni 1, 27 BA, t. I, 469; PL 42, 558).

29. Op. cit. $1,1 \mathrm{BA}$, t. I, 441; PL 42, 551.

30. De lib. arb. 3,20, $54 \mathrm{PL} 32,1270 ; \mathrm{J}$. MOREAU, Le temps et la création selon saint Augustin: "Giornale di Metafisica", 20 (1965), 276-290.

31. De natura boni $1,25 \mathrm{PL} 42,559$. 
miurge platonien. On n'attribuait au Démiurge que le rôle de transformer le chaos en structures finales et ordonnées. Le Créateur chrétien donne à l'univers le plus précieux des biens: l'existence ${ }^{32}$. Le Summum Bonum est tout d'abord source de l'existence, et en second lieu source d'autres perfections. Le bien créé n'est pas permanent quant à son existence, car il a été appelé à l'existence du néant antérieur. Dieu, en créant l'univers, ne lui a pas donné d'existence propre permanente, mais une existence (fragile), toujours proche du néant. Il en résulte un caractère éphemère des créatures se manifestant par leur passage au (non-être», à la (non-perfection».

Il y a encore une autre differénce, entre le créationnisme augustinien et l'émanationnisme néo-platonicien. Les Ennéades autorisent à parler d'un déterminisme du processus d'émanation ${ }^{33}$. St. Augustin cependant constate l'entière liberté de l'acte créateur: Dieu nous a créés, car Il le voulait ${ }^{34}$. Il ne Lui manquait aucune perfection et c'est pourquoi, en nous donnant l'existence, Il l'a fait volontairement et par bienveillance. Le même motif - la bonté- fait que Dieu conserve constantement l'existence du monde créé par Lui ${ }^{35}$. La conservatio in esse se trouve également dans le néo-platonisme, mais possède alors une tout autre ambiance, surtout par son affinité à l'émanationnisme panthéisant ${ }^{36}$.

Jusqu'à présent, l'analyse de la théorie augustinienne de l'acte créateur démontre, entre l'évêque d'Hippone et Plotin, une divergence d'opinions considérable quant au problème de la participation au bien. Les oeuvres du penseur chrétien contiennent cependant des formulations que témoignent de l'influence de la conception neo-platonicienne du Bien sur la conception augustinienne du Summum Bonum. Cela a été remarqué par A. Gardeil; à son avis, "St. Augustin, en suivant Platon, attribue la création des choses à leur participation aux idées de Dieu) ${ }^{37}$. Gilson exprime également la convinction que l'Absolu, chez Augustin, est incontestablement le Dieu chrétien - donneur d'existence-, mais que c'est simultanément le

\footnotetext{
32. De civ. Dei 12, 2 PL 41, 350; TH. ROSER, "Emanation and Creation. The Doctrine of Plotinos and Augustinus on the radical Origin of the Universse: New Scholasticism", 19 (1945), 85-116.

33. Voir: PLOTIN, En. II, 9, 3; V, 5, 9

34. "Quia voluit, fecit" (ad Orosium c. Priscill. et Origen. 1, 2 PL 42,671); "Nulla necessitate [...] sed sola bonitate fecisse" (De civ. Dei 11, 24 PL 41, 338).

35. "Usque nunc operatur Deus" (De Gen. ad litt. 5, 20 PL 34,335); voir op. cit. 4, 12, 23 PL 34,305; De civ. Dei 12, 17.

36. Voir: J. JASINOWSKI, 0 istocie neoplatonizmu (De la substance du neo-platonisme) "Przeglad Filozoficzny", 20 (1917), 232 ss.

37. A. GARDEIL, La structure de l'âme et l'expérience mystique, París 1927, t. II, 319.
} 
Dieu créant l'être au sens de Platon ${ }^{38}$. L'influence de Platon et de Plotin se manifeste par l'usage de la terminologie. Dans le traité De libero arbitrio et dans les Confessions, le texte est caractéristique lorsqu'il décrit l'acte créateur comme formatio, c'est-à-dire comme processus qui met de l'ordre dans le monde existant à l'état de chaos et lui atribue finalité et sens ${ }^{39}$. Dieu apparaît dans le rôle de Forme suprême et immutable et les choses participent à ses perfections par l'acte de formation intérieure. Une telle interprétation de l'acte créateur est incontestablement un appauvrissement de celui-ci, mais il s'agit du niveau des formulations verbales, pas toujours hereuses. Il est certain cependant que cla doctrine de l'acte créateur de l'univers dans le temps, développée par Augustin, est entièrement chrétienne) ${ }^{40}$.

b. L'expansion du Summum Bonum dans l'acception de St. Augustin, se faisat également au niveau de la causalité exemplaire. C'est celle-ci qui, proprement parler, jouit un rôle essentiel dans la relation unissant les biens créés avec le Bien Suprême. Cela résultait de l'influence de Platon qui considérait les idées comme les modèles primordiaux immatériels des espèces ${ }^{41}$. Les idées étaient liées à l'Absolu, mais elles en différaient réellement ${ }^{22}$. La théorie des idées a été incluse par Plotin dans son émanationnisme. La siège des idées était le Nus, interprêté comme la première et la plus haute émanation de l'Unité. Les idées forment le kosmos noetós dont le monde visible est le réflet ${ }^{43}$.

St. Augustin a assimilé les deux théories en tâchant de les faire concorder avec la théorie de la création. Thomas d'Aquin juge positivement cet essai de synthèse et il constate la transformation de la théorie des idées platonienne en théorie exemplariste ainsi que son union à la conception d'illumination de la raison humaine par Dieu ${ }^{44}$. La théorie augustinienne des idées, bien qu'elle ait de nombreux élé-

\footnotetext{
38. E. GILSON, Le Thomisme, Paris 1947, 193-196.

39. De lib, arb. 2, 17, 45, PL 32,1265; Confes. 13, 2, 2 PL 32,845.

40. Th. RUSER, Emanation and Creation: "New Scholasticism" 19 (1945), 112.

41. Voir: PLATON, Le Banquet $211 \mathrm{a}-\mathrm{b}$; la République VI, 505a-507b.

42. II interprète Platon ainsi: R. LORIAUX, L'être et l'idée selon Platon: "Revue Néoscholastique de Louvain": 50 (1952), 5-55; J. SOUILHE, La philosophie religieuse de Platon: "Archives de Philosophie", 26 (1963), 387-436.

43. Voir: PLOTIN, En I, 3, 1; VI, 7, 25; VI, 7, 36

44. Voir: ST. THOMAS D'AQUIN, De spir. cr. 10, ad 8. Nous ne discutons pas, dans la présente dissertation, le problème compliqué de l'illuminisme augustinien. Voir: T. ALESANCO, Metafisica y gnoseologia del mundo inteligible según S. Agustín. En torno a la teoría aqustiniana de la iluminación: "Augustinus", 13 (1938), 9-36; R. H. NASH, The Light of the Mind, Lexington 1969.
} 
ments convergents, diffère cependant de la théorie de Platon d'une manière essentielle. Pour Platon, les idées étaient les modèles primordiaux des espèces, tandis que pour St. Augustin, elles étaient les modèles des choses individuelles. La seconde différence consiste en ce que le platonicien chrétien a localisé les idées dans l'intellect de Dieu. Les idées modèles existant en Dieu forment le monde qui, du côté de la nature divine est simple, et du côté des "copies» (réflets) comple$\mathrm{xe}^{45}$. Les idées modèles des choses, en tant qu'idées de l'intellect divin, participent à son éternité et à son immutabilité. Elles sont (des formes ou des raisons constantes et immutables des choses (...) éternelles (...) qui se trouvent dans l'intellect de Dieu» ${ }^{46}$. Augustin appelait les idées modèles également rationes seminales, éventuellement causes des choses ${ }^{47}$. Elles possèdent leur réalité et leur dynamisme: ce sont les pensées éternelles de Dieu et qui, en temps utile et par l"omnipotence du Créateur, appelent les choses à l'existence.

Maintes fois, St. Augustin exploitait la théorie de causalité exemplaire en expliquant les relations unissant le Bien Suprềme au monde des valeurs visuelles. A son avis, ce monde de participation au Bien absolu, lié à la théorie mimesis de Platon, fait le mieux valoir sa nature. Alors même qu'il parle de causalité efficiente, il se reporte souvent à la causalité exemplaire ${ }^{48}$.

D'habitude, la littérature concernant la théorie augustinienne de participation, insiste sur ses connexions avec Platon. Ce rétrécissement du domaine des influences n'est pas juste pour la théorie du bien, dans laquelle les filiations à Plotin apparaissent en particulier. L'auteur des Ennéades, en indiquant le gradualisme des biens, voit ses origines dans la manière dont les êtres - spirituels ou matériels approchent les: "Bien - idée»" ${ }^{49}$. Leur valeur augmente dans la mesure de leur purification des entraves matérielles, ce qui équivaut à une idéalisation graduée. Augustin accepte la structure gradualiste des valeurs. Tous les biens participent au Bien Suprême, mais pas tous au

\footnotetext{
45. "Quia igitur unum Verbum Dei est, per quod facta sunt omnia, quod est incommutabilis veritas, ibi principaliter atque incommutabiliter sunt omnia simul; non solum quae nunc sun in hac universa creatura, verum etiam quae fuerunt et quae futura sunt" Trin. $4,1,3$ PL 42,888; voir: De civ. Dei 12,8 PL 41,368 .

46. Div. quaest. oct. tr. $46,2 \mathrm{PL} 40,30$.

47. "Et apud te rerum omnium instabilium stant causae; et rerum omnium mutabilium immutabiles manent origines; et omnium irrationabilium et temporalium sempiternae vivunt rationes" Confes. 1, 6, 9, PL 32,664; "Una sapientia est, [...] in qua sunt omnos invisibilium atque incommutabiles rationes rerum, etiam visibilium et mutabilium" (De civ. Dei 11, 10, 3 PL 41,327).

48. "Et fecit Deus omnia valde bona [...] Multis enim modis dici res possunt similes Deo" (Div. quaest. oct. tr. 51, 2 PL 40, 32).

49. PLOTIN, En. VI, 7, 25; VI, 7, 28.
} 
même degré ${ }^{50}$. Le degré de similitude au Bien primordial est la mesure du bien ontique des créatures ${ }^{51}$. Ainsi, les minéraux ressemblent à Dieu par le fait d'exister, les organismes vivants par la possession de la vie, les hommes par la possession de la raison et de la volonté.

L'union de la conception du Summum Bonum avec la causalité modèle est bien présentée dans le passage suivant du traité De Trinitate: "Donc, dans la mesure où tout être est bon, il possède, encore que très lointane, une certaine ressemblance avec le souverain Bien' " ${ }^{52}$. Pour Augustin, le fondement du bien des créatures se trouve avant tout dans le fait qu'elles reflèt e $n t$ en elles les perfections du Bien Suprême. Il s'agit donc expressément de causalité exemplaire bien que liée à la causalité efficiente. Toute créature est une image reflétée du bien divin en conformité avec sa nature générique; c'est la similitude de la créature au Créateur. $\mathrm{Si}$ un être doué de raison désire s'assimiler à Dieu d'une manière contraire à la nature, il tombe dans le mal moral.

En expliquant le fait de participation des êtres au Bien Suprême, Augustin emploie une terminologie liée à la théorie platonienne de réflexion. Plotin expliquait le plus souvent la relation de l'Unité au monde visible par les notions: modèle-copies ${ }^{53}$. L'Unité est le seul bien authentique, plein et idéal; toute autre chose n'est que son réflet, presque son ombre. Fréquemment, l'évêque d'Hippone a recouru à de telles expressions, en affirmant une participatio similitudinis entre les créatures et le Créateur. Dieu est le bien absolu et suprême, les créatures "existent dans son éternité, sa vérité et sa charité) " ${ }^{54}$. Parmi tous les êtres, 1'homme, doué de raison, de mémoire et de volonté, est la réflet le plus parfait de la perfection du Bien Suprême, l'imago Dei ${ }^{55}$. Les autres êtres ne sont qu'ombre ou trace de Dieu; l'homme est Son image. En caractérisant la causalité exemplaire du Summum Bonum, St. Augustin est lié, incontestablement, à Platon et à Plotin. Toutefois, il a modifié essentiellement leurs théories, en les conciliant avec le créationnisme chrétien. Jamais il ne mettait en

\footnotetext{
50. Voir: De mor. Man. 2, 4, 6 PL 32, 1347.

51. "Nemo enim dubitat quod sit ipse primitus bonus. Multis enim modis dici res possunt similes Deo: aliae secundum virtutem et sapientiam factae, [...] aliae in quantum solum vivunt, quia ilie summe ef primitus vivit, aliae in quantum sunt, quia ille summe et primitus est" Div. quaest. oct. tr. 51,2 PL 40,32 ).

52. Trin. 11, 5, 8, BA, t. XVI, 185; PL 42, 991.

53. En III 5,$1 ; \mathrm{V}, 3,13 ; \mathrm{VI}, 7,18$

54. De civ. Dei 12, 1, 2 BA, t. XXXV. Trad. par G. Combès, Paris 1959, 151; PL 41, 349

55. C. Faust. Man, 22, 27 PL 42,418
} 
doute la réalité des bien créés. Il voyait en eux des(copies», des (effigies) du Bien Suprême, mais la similitude des creatures au Createur était réalisée au niveau de l'existence réelle. Les créatures, et avant tout, les êtres raisonnables et libres sont, de par leur nature et du fait d'exister, similaires à Dieu, bien absolu.

c. Dieu, en tant que Summum Bonum, est également la cause finale de l'univers. Dieu est non seulement le Créateur et la cause exemplaire du monde, mais encore la fin, le but suprême de celui-ci, et pour les créatures raisonnables, il est également bonheur, L'évêque d'Hippone désignait du nom de fin la cause, le motif de l'activité ${ }^{56}$. La fin n'a d'attrait que si elle présente pour le sujet agissant un bien authentique ou du moins apparent. Tout d'abord, le bien suscite l'intérêt, ensuite l'amour et enfin, il incite à l'action pour le conquérir.

Le plus souvent, ce n'est ni dans l'extrême égoïsme, ni dans l'altruisme pur que se trouve le moteur de l'activité humaine, mais dans le réflexe si naturel du coeur humain qu'est le désir d'être heureux. Ce désir éveille l'amour du bien. Les deux phénomènes - la recherche du bonheur et l'amour du bien - sont aussi universels que l'humanité ${ }^{57}$. Sous l'influence du néo-platonisme ainsi que de la Révélation chrétienne, Augustin voit dans l'amour le facteur décisif de la vie émotionelle et volitionnelle de l'homme. D'autres actes émotionnels, comme la joie, la tristesse, la déception, ne sont que manifestations de l'amour. L'élément d'amour doit également dominer dans la vie morale et religieuse; de là, la maxime augustinienne: "dilige et quod vis $f a c){ }^{58}$. Toute la vie psychique devrait se centraliser autour de l'amour du bien. Un amour primitif apparaît chez les animaux sous la forme de l'instinct et même chez les plantes et les êtres inanimés, sous forme de (l'amour naturel» qui se manifeste par le désir de conserver son existence ${ }^{59}$.

Tous les hommes désirent la bonheur et aiment le bien. Les uns voient dans la cumulation de bien matériels, les autres dans le plaisir de vivre et les émotions, d'autres dans la vie active, dans le service du prochain, et d'autres encore dans la vie contemplative, c'est-à-di-

56. C. Adver. Leg. et Proph. 2, 8, 26 PL 42, 653.

57. "Nemo est qui non amat" (Sermo 34, 2 P'-38, 210); voir: A. BECKER, De l'instinct du bonheur à l'extase de la béatitude, Paris 1967, passim.

58. In ep. Joan. ad Part. 7, 8 PL 35, 2033; G. HULTGREN, Le commandẹment d'amour chez Augustin, Paris 1939, passim.

59. Trin. $8,3,4$ PL $42,949-950$. 
re dans la recherche de la vérité. L'évêque d'Hippone ne blâme point les valeurs accidentelles ni même celles matèrielles et sensitives. Il ne défend pas de les apprécier ni même de les aimer. Cependant, il existe un ordo amoris objectif que l'on doit respecter. Un plus grand amour est dû aux biens supérieurs par leur nature, permanents dans leur existence, et plus universels quant à leur domaine; ce sont les biens spirituels et, en particulier, les biens moraux. Augustin en tire la conclusion suivante: "Celui qui aime l'existence, qu'il aime donc ce qui existe toujours) ${ }^{60}$. Il n'y a pas de choses mauvaises par leur nature, il peut $y$ avoir toutefois (un mauvais usage du bien) ${ }^{61}$. Cela arrive lorsque l'homme renverse l'ordre naturel en mettant les biens accidentels les créatures, à la place du Bien immutable, du Créateur. Dieu est le Bien Suprême mais invisible; les créatures sont des biens relatifs mais visibles. La fascination inconsidérée par les biens partiels conduit à l'ignorance de la valeur intégrale, de Dieu. Et cependant, l'homme "qui se réjouit du Bien Suprême») est celui qui atteint le bonheur plein et véritable ${ }^{62}$.

Le désir du bonheur est la force motrice de la vie humaine. Le bonheur est accessible par le bien. Le bien qui incite à l'amour devient la fin de notre activité. Augustin distingue deux types de biens: les uns sont employés par l'homme, les autres le mènent à la perfection. Les biens matériels (habits, nourriture, habitation) possèdent un caractère de consommation, mais les valeurs spirituelles, surtout morales et religieuses, nos donnent une formation intérieure ${ }^{63}$. C'est ainsi qu'il y a lieu de constater que les créatures ne sont que mo y e $n$ et que Dieu seul en tant que Summum Bonum, est la fin suprême de la vie humaine.

L'amour est l'équivalent subjectif du bien. Dans De Trinitate, nous lisons: "Qu'est donc que l'amour ou charité, (...) sinon l'amour du Bien? Mais l'amour vient de quelqu'un qui aime, et par l'amour quelque chose est aimé. Et voici trois choses: celui qui aime, ce qui est aimé, et l'amour même. Qu'est-ce donc que l'amour, sinon une certaine vie qui unit deux êtres ou tend à les unir: celui qui aime et

\footnotetext{
60. De lib. arb. 3, 7, 21 BA t. VI. Trad par F. J. Thonnard, Paris 1952, 365; PL 32, 1281.

61. "Malum est male uti bono" (De natura boni 1, 36 PL 42, 562).

62. De lib arb. 2, 13, 36 BA, t. VI, 285; PL 32, 1260.

63. "Hic [Deus] est finis amoris nostri; finis quo perficiamur non quo consummamur" (Sermo 53, 6, 6 PL 38, 366). Voir: A. DI GIOVANNI, L'inquietudine dell'anima. La dottrina dell'amore nelle Confessioni di sant'Agostino, Roma 1964, passim.
} 
l'être qui est aimé?" " ${ }^{64}$. Augustin a remarqué judicieusement que le principe de communion, voire d'une identification partielle, regit $l^{\prime}$ amour. La cupiditas égoïste désire prendre pour soi, mais l'authentique caritas désire partager la perfection qu'elle possède, avec une autre personne.

L'intensité de l'acte d'amour doit s'accroître en mesure de la valeur objective de l'objet aimé. L'amour des choses terrestres est autre que l'amour de la Valeur Immutable. Il faut aimer Dieu de tout coeur: la mesure de Son amour est l'amour sans bornes pour Lui. C'est l'amour pur: amor, caritas, dilectio, qui comporte la pleine communion de l'homme avec Dieu ${ }^{65}$. Les créatures doivent égalment faire l'objet de notre amour, mais elles ne sont qu'un moyen qui sert à atteindre le but suprême et ne pouvent en elles-mêmes être l'objectif final ${ }^{66}$. Un amour exagéré des créatures et qui abolit la hiérarchie naturelle des valeurs, n'est pas la vrais caritas, il n'est qu'une cupiditas égoïste ${ }^{67}$.

L'amour du bien donne joie, contentement, bonheur. La mesure de ce bonheur dérive de la richesse de l'objet aimé. Le bien mutable donne un bonheur éphémère et partiel. Le Bien absolu sera la source d'une béatitude complète et éternelle. Dans son commentaire au psaume 26, St. Augustin affirme que (si les biens qui ne sont pas des entiers - les biens mutables ne sont pas des biens autonomes-, nous remplissent de bonheur, quelle sera donc la contemplation du Bien immuable, éternel, demeurant toujours le même? $){ }^{68}$. Dieu, Summum Bonum objetif, est également summum bonum pour l'homme. Il comporte la plénitude des valeurs spirituelles qui seront dans la vie future la source d'une éternelle béatitude.

St. Augustin tâche de définir la nature de la contemplation de Dieu. Dans le traité De libero arbitrio, l'authentique bonheur est appelé par lui ("ve $\mathrm{r}$ i té dans laquelle le bien suprême est aperçu) ${ }^{69}$. Une parole extraite des Confessions en est le complément: "La vie

64. Trin. 8, 10, $14 \mathrm{BA}$, t. XVI, 71; PL 42, 960; M. HUFTIER, La charité dans l'ẹnsęignement de saint Augustin Paris 1960, passim.

65. De mor. Eccl. 1, 22, 40 PL 32, 1228-1229.

66. "Solo Deo fruendum [...] caeteris autem utendum est" (De Doct. chr. 22, 20 PL. 34, 26); voir: N. HARTMANN, Ordo amoris. Zur augustinischen Wesensbestimmung des Sittlichen: "Wissenschaft und Weisheit". 18 (1955), 1-23.

34.

67. Enar. in ps. 9 PL 36, 124; De Doct. chr. 3, 10, 16 PL 34, 72. Voir: PLOTIN, En. VI, 7,

68. Enar. in ps. 26, $8 \mathrm{PL} 36,203$; voir: R. HOLTE, Béatitude et Sagesse. Saint Augustin et le problème de la fin de l'homme dans la philosophle ancienne, Paris 1962, 221-270.

69. De lib. arb. 2, 9, 26 BA, t. VI, 261; PL 32, 1254. 
heureuse est la joie de la verité) ${ }^{70}$. Une pleine contemplation de Dieu exige des actes cognitifs de l'intellect ainsi que des actes de volition vers le bien. Les deux éléments sont indispensables, puisque la connaissance de la verité est condition de l'amour, et celui-ci, â son tour, enrichit les actes cognitifs.

Théoriquement, le problème de la relation réciproque de la connaissance et de l'amour dans la contemplation de Dieu, a été résolu par l'évêque d'Hippone dans ses oeuvres écrites avec mesure. Dans la pratique cependant, et comme il professait le volontarisme, St. Augustin a accentué le rôle des actes à tendance. Le Bien Suprême n'est éprouvé d'une manière adéquate que dans les actes de prédilection et d'amour, et c'est ainsi que "celui qui n'a pas d'amour, ne connaît point Dieu, car Dieu est amour) " ${ }^{71}$. "De quoi donc, sinon de Dieu, est rempli celui qui est plein d'amour' ${ }^{72}$. La vraie expérience, voire la connaissance de Dieu, sont conditionnées par les actes d'amour. L' amour est l'acte qui nous met en possession du bien désiré. L'intellect réduit partiellement en atomes l'objet dont il prend connaissance; l'amour le conçoit d'une manière intégrale. La connaissance spéculative est une acception indirecte et extérieure du bien, tandis que l'acte d'amour nous fait personnellement éprouver la valeur. L'evêque d'Hippone en tire la conclusion suivante: "Nous ne pouvons appartenir à Dieu autrement que par la prédilection, la dilection, l' amour) ${ }^{73}$.

\section{Katolicki Uniwersytet Lubelski Stanislas Kowalczyk}

70. Confes. 10, 23, 33 BA, t. XIV, 201; PL 32, 793. Voir: F. CAYRE, La contemplation augustinienne, Paris 1954, 133-237.

71. Trin. 15, 17, $31 \mathrm{BA}$, t. XVI, 509; PL 42, 1082; voir: J. MUNOZ, -San Augustin, iniciador del 'intulcionismo emocional'?": "Pensamiento" 10 (1954), 455-487.

72. Trin. 8, 8, $12 \mathrm{BA}$, t. XVI, 63; PL 42, 958. Voir: Confes. 7, 10, 16 PL 32, 599; De mor. Eccl. 1, 25, $47 \mathrm{PL} 32,1331$; A. MAXSEIN, Philosophia cordis. Das Wesen der Personnalität bei Augustin, Salzburg 1966, passim.

73. De mor. Eccl. 14, 24 PL 32, 1322. 